Unfallchirurg 2016 $119: 262-263$

DOI 10.1007/s00113-016-0157-5

Online publiziert: 22. März 2016

๑) Springer-Verlag Berlin Heidelberg 2016

CrossMark

\author{
P. Biberthaler \\ Klinik und Poliklinik für Unfallchirurgie, Klinikum rechts der Isar, Technische Universität München, \\ München, Deutschland
}

\title{
Periprothetische Frakturen Teil 2
}

Sehr geehrte Leserinnen und Leser,

wie schon angekündigt, darf ich Ihnen heute den 2. Teil des Leitthemas Periprothetische Fraktur vorstellen. Nachdem in der letzten Ausgabe die untere Extremität ausführlich behandelt wurde, soll in dieser Ausgabe auch die obere Extremität beleuchtet werden. Obwohl die Gesamtzahl der Prothesen an der oberen Extremität deutlich geringer ist im Vergleich zur unteren Extremität, so sind die Versorgungsstrategien mindestens so komplex. Dies betrifft unter anderem die zunehmende Frakturendoprothetik am Schultergelenk. Hier konnten moderne Konzepte der primären inversen Prothetik sehr gute frühe und mittelfristige Ergebnisse erzielen, sodass die Frage nach der optimalen Therapie bei potenziellen Frakturen z. B. auf glenoidaler Seite mehr Relevanz erhält. Zu diesem Thema gibt es nur wenige systematische Untersuchungen, sodass der Beitrag in Der Unfallchirurg sicherlich einer der Referenzartikel werden wird. Aber auch die Weiterentwicklungen im Bereich der Ellenbogenprothesen erfordern neue Denkweisen der optimalen therapeutischen Strategie. Die spezifischen Anforderungen an die Osteosynthese werden in den folgenden Beiträgen sehr präzise und übersichtlich zusammengefasst. Hier haben sich in der jüngeren Vergangenheit einige ganz wesentliche Entwicklungen gezeigt, die wir Ihnen gerne näherbringen möchten. Gerade in diesen Situationen der kritischen Blutversorgung bei einliegenden intramedullären Schäften kommt der optimalen OP-Technik ganz entscheidende Bedeutung zu. Hier hat sich gezeigt, dass die klassischen Kriterien wie akribische Reposition, weichteilschonende Präparation etc. noch viel mehr ihre Gültigkeit bewiesen haben.
Somit liegt mit dieser und der vergangenen Ausgabe ein umfangreiches Update für die Behandlung dieser zunehmend relevanten Entität vor, welches sicherlich als Nachschlagewerk Einzug in viele persönliche Bibliotheken nehmen wird.

Ich wünsche Ihnen viel Erfolg für die Behandlung Ihrer Patienten und hoffe, dass wir Ihnen hierfür die neuesten Kenntnisse zur Verfügung stellen konnten.

Herzliche Grüße

Ihr

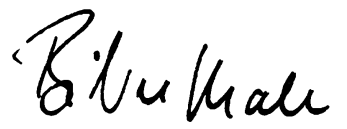

Prof. Dr. Peter Biberthaler

\section{Korrespondenzadresse}

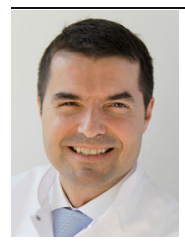

Prof. Dr. P. Biberthaler

Klinik und Poliklinik für Unfallchirurgie, Klinikum rechts der Isar, Technische Universität München Ismaninger Str. 22, 81675 München, Deutschland Peter.Biberthaler@mri.tum.de 
Hier steht eine Anzeige.

算 Springer 